\title{
Autonomic Nervous System Neoplasm
}

National Cancer Institute

\section{Source}

National Cancer Institute. Autonomic Nervous System Neoplasm. NCI Thesaurus. Code C5112.

Benign and malignant neoplasms which arise from or directly involve the central or peripheral elements of the autonomic nervous system. 